\title{
FRACTIONAL INTEGRAL OPERATOR AND STATISTICAL DISTRIBUTION
}

\author{
MRIDULA GARG AND MAHESH KUMAR GUPTA
}

\begin{abstract}
The present paper deals with a generalized fractional integral operator involving the well-known Fox's H-function. Here, we first obtain the images of certain special functions under this operator and then apply them to the study of a generalized form of a finite statistical distribution. Thus, we obain the distribution function, characteristic function and mathematical expectation of a function for this statistical distribution. We also mention certain, known particular cases of our findings.
\end{abstract}

\section{Introduction and Definitions}

The aim of this paper is to study the application of the following fractional integral operator $[9, \mathrm{p} .2]$ to certain statistical distributions.

$$
\begin{aligned}
I_{o, x}^{\sigma}[f(x)] & =I_{o, x, a_{j}, \alpha_{j}, b_{j}, \beta_{j}}^{\sigma, M, N, P,{ }_{j}}[f(x)] \\
& =\frac{x^{-\sigma}}{\Gamma(\sigma)} \int_{0}^{x}(x-t)^{\sigma-1} H_{P, Q}^{M, N}\left[z\left(1-\frac{t}{x}\right) \mid \begin{array}{c}
\left(a_{j}, \alpha_{j}\right)_{1, P} \\
\left(b_{j}, \beta_{j}\right)_{1, Q}
\end{array}\right] f(t) d t
\end{aligned}
$$

where $\sigma>0$ and $H_{P, Q}^{M, N}[x]$ stands for the well-known Fox's $\mathbb{H}$-function [3] defined by [14, p.10, eq.(2.1.1)].

In the sequel we shall also use the multivariable $\mathbb{H}$-function introduced by Srivastava and Panda [17] which will be represented in the contracted form used in the book [14, p.251, eq.(C.1)].

For the definitions, various special cases and other details of Fox's H-function and the multivariable $\mathbb{H}$-function, we refer the above mentioned book.

It may be remarked here that all the Greek letters occurring in these functions are assumed to be positive real numbers for standardization purpose; the definitions of these functions will, however, be meaningful even if some of these quantities are zero. Again, it is assumed throughout the present work that these functions always satisfy the appropriate conditions of convergence [14, pp.12-13, eqns.(2.2.1), (2.2.11) and pp.252-253,

Received January 6, 1997, Revised March 25, 1997.

1991 Mathematics Subject Classification. 33C40, 26 A33.

Key words and phrases. Fractional integral opetator, Fox's H-function, multivariable Hfunction, a class of polynomials, finite statistical distribution. 
eqns.(C.4-C.6, respectively]. Also let $S_{V}^{U}[x]$ denote the class of polynomials introduced by Srivastava [11, p.1, eq.(1)] as follows:

$$
S_{V}^{U}[x]=\sum_{K=0}^{[V / U]} \frac{(-V)_{U K} A_{V, K} x^{K}}{K !} \quad(V=0,1,2, \ldots)
$$

where $U$ is an arbitrary positive integer and the coefficients $A_{V, K}(V, K \geq 0)$ are arbitrary constants, real or complex. On suitably specializing the coefficients $A_{V, K}, S_{V}^{U}[x]$ yields a number of known polynomials as its special cases [18, pp.158-161].

\section{Images Under Fractional Integral Operator (1.1)}

Here, we obain images of some elementary functions and Fox's H-functions under the fractional integral operator defined by (1.1). The results are given below:

Result $\mathbb{1}$. If $\sigma>0, k+1>0, \sigma+\min _{1 \leq j \leq M}\left(b_{j} / \beta_{j}\right)>0$ and $|x / a|<1$, then

$$
\begin{aligned}
I_{0, x}^{\sigma}\left[x^{k}(x+a)^{-c}\right] & =\frac{a^{-c} x^{k}}{\Gamma(c) \Gamma(\sigma)} H_{0,1: P+1, Q ; 2,1}^{0,0: M . N+1 ; 1,2} \\
& {\left[\begin{array}{l}
z \\
z / a \mid(-k-\sigma: 1,1):\left(b_{j}, \beta_{j}\right)_{1, Q}
\end{array}\right] }
\end{aligned}
$$

Proof. Using the definition (1.1) in left hand side of (2.1) and subsituting $t=x v$ in the integral so obtained, expressing the Fox's H-function in the series form [14, p.12, eq.(2.2.4)] and interchanging the order of summation and integration, we arrive at the right-hand side of (2.1) after a little simplification.

Taking $c=0$ in the above result, we arrive at the following useful formula after some simplification.

$$
I_{o, x}^{\sigma}\left[x^{k}\right]=\frac{x^{k} \Gamma(1+k)}{\Gamma(\sigma)} H_{P+1, Q+1}^{M, N+1}\left[z \mid \begin{array}{l}
(1-\sigma, 1),\left(a_{j}, \alpha_{j}\right)_{1, P} \\
\left(b_{j}, \beta_{j}\right)_{1, Q},(-\sigma-k, 1)
\end{array}\right]
$$

Result 2. If $\sigma>0, k+\sum_{i=1}^{r} \min _{1 \leq j \leq M_{i}}\left(b_{j}^{(i)} / \beta_{j}^{(i)}\right)+1>0$ and $\sigma+\min _{1 \leq j \leq M}\left(b_{j} / \beta_{j}\right)>0$ $(i=1, \ldots, r)$, then

$$
I_{o, x}^{\sigma}\left(x^{k} \prod_{i=1}^{r}\left\{H_{P_{i}, Q_{i}}^{M_{i}, N_{i}}\left[z_{i} x \mid \begin{array}{l}
\left(a_{j}^{(i)}, \alpha_{j}^{(i)}\right)_{1, P_{i}} \\
\left(b_{j}^{(i)}, \beta_{j}^{(i)}\right)_{1, Q_{i}}
\end{array}\right]\right\}\right)
$$




$$
\begin{aligned}
& =\frac{x^{k}}{\Gamma(\sigma)} H_{1,1: P_{1}, Q_{1} ; \ldots ; P_{r}, Q_{r} ; P+1, Q}^{0,1: M_{1}, N_{1} ; M_{r}, N_{r} ; M, N+1}\left[\begin{array}{l}
z_{1} x \mid(\underbrace{1, \ldots, 1}_{(r)}, 0): \\
\vdots \\
z_{r} x \\
z
\end{array}\right](-k-\sigma: \underbrace{1, \ldots, 1}_{(r+1)}): \\
& \left.\left(a_{j}^{(1)}, \alpha_{j}^{(1)}\right)_{1, P_{1}} ; \cdots ;\left(a_{j}^{(r)}, \alpha_{j}^{(r)}\right)_{1, P_{r}} ;(1-\sigma, 1),\left(a_{j}, \alpha_{j}\right)_{1, P}\right] \\
& \left.\left(b_{j}^{(1)}, \beta_{j}^{(1)}\right)_{1, Q_{1}} ; \cdots ;\left(b_{j}^{(r)}, \beta_{j}^{(r)}\right)_{1, Q_{r}} ;\left(b_{j}, \beta_{j}\right)_{1, Q}\right]
\end{aligned}
$$

Proof. Expressing each of Fox's H-function occurring in the left hand side of (2.4) in the series form, changing the order of integration and summations, invoking the result (2.3), we arrive at the right hand side of (2.4) after a little simplification.

Result 3. If $\sigma>0, \sigma+\min _{1 \leq j \leq M}\left(\frac{b_{j}}{\beta_{j}}\right)>0, k+\rho \mathbb{K}+\sum_{i=1}^{r} \min _{1 \leq j \leq M_{i}}\left(b_{j}^{(i)} / \beta_{j}^{(i)}+1>0\right.$, $i=1, \ldots, r$ and $K=0,1, \ldots,\left[\frac{V}{U}\right]$ then

$$
\begin{aligned}
& I_{o, x}^{\sigma}\left(x^{k} e^{\alpha x} \prod_{i=1}^{r}\left\{H_{P_{i}, Q_{i}}^{M_{i}, N_{i}}\left[z_{i} x \mid \begin{array}{l}
\left(a_{j}^{(i)}, \alpha_{j}^{(i)}\right)_{1, P_{i}} \\
\left(b_{j}^{(i)}, \beta_{j}^{(i)}\right)_{1, Q_{i}}
\end{array}\right]\right\} \times S_{V}^{U}\left[y x^{\rho}\right]\right) \\
& =\sum_{K=0}^{[V / U]} \frac{(-V)_{U K} A_{V, K} y^{K} x^{k+\rho K}}{\mathbb{K} ! \Gamma(\sigma)} H_{1,1: P_{1}, Q_{1} ; \ldots ; P_{r}, Q_{r} ; 0,1 ; P+1, Q}^{0,1: M_{1}, N_{1}, \ldots ; M_{r}, N_{r} ; 1,0 ; M, N+1}\left[\begin{array}{l}
z_{1} x \\
\vdots \\
z_{r} x \\
-\alpha x \\
z
\end{array} \mid\right. \\
& (-k-\rho \mathbb{K}: \underbrace{\mathbb{1}, \ldots, 1,0)}_{(r+1)}:\left(a_{j}^{(1)}, \alpha_{j}^{(1)}\right)_{1, P_{1}} ; \cdots ;\left(a_{j}^{(r)}, \alpha_{j}^{(r)}\right)_{1, P_{r}} \\
& (-k-\sigma-\rho \mathbb{K}: \underbrace{1, \ldots, 1}_{(r+2)}):\left(b_{j}^{(1)}, \beta_{j}^{(1)}\right)_{1, Q_{1}} ; \cdots ;\left(b_{j}^{(r)}, \beta_{j}^{(r)}\right)_{1, Q_{r}} \\
& ;--;(\mathbb{1}-\sigma, 1),\left(a_{j}, \alpha_{j}\right)_{1, P} \\
& ;(0, \mathbb{1}) ;\left(b_{j}, \beta_{j}\right)_{1, Q}
\end{aligned}
$$

The proof of the above result can be carried out on the lines similar to those of Result 2 .

It may be mentioned here that the result (3) can be manipulated to yield Results 1 and 2. Also it yields the results given recently by Saxena [7, p.208, eq. $(5,2,10)]$ and Srivastava and Goyal [13, p.649, eqns.(3.6) and (3.7)] on proper specialization.

Recently Srivastava and Hussian [15] and Srivastava and et.al. [12, p.570, eq.(3.10)] 
have also obtained the results involving the fractional calculus of $\mathrm{H}$-function of several variables.

\section{Finite Statistical Distribution}

Let us define a family of distributions having the probability density function of the following form. For $\sigma>0, h \neq k$

$$
f(x)=\left\{\begin{array}{l}
\frac{1}{\Delta}(x-h)^{p-1}(k-x)^{\sigma-1} H_{P, Q}^{M, N} \\
\left.\times \prod_{i=1}^{r}\left\{H_{P_{i}, Q_{i}}^{M_{i}, N_{i}}\left[\begin{array}{l}
z-x \\
k-h
\end{array} \mid \begin{array}{l}
\left.(x-h) \mid \begin{array}{l}
\left(a_{j}, \alpha_{j}\right)_{1, P} \\
\left(b_{j}, \beta_{j}\right)_{1, Q}
\end{array}\right] \\
0, \text { elsewhere }
\end{array}\right] \begin{array}{c}
\left(a_{j}^{(i)}, \alpha_{j}^{(i)}\right)_{1, P_{i}} \\
\left(b_{j}^{(i)}, \beta_{j}^{(i)}\right)_{1, Q_{i}}
\end{array}\right]\right\} \text { for } h \leq x \leq k
\end{array}\right.
$$

where

$$
\begin{aligned}
& \Delta=(k-h)^{p+\sigma-1} H_{1,1: P_{1}, Q_{1} ; \ldots: P_{r}, Q_{r}: P+1, Q}^{0,1: M_{1}, N_{1}: \ldots: M_{r}, N_{r}: M, N+1}\left[\begin{array}{l}
z_{1}(k-h) \\
\vdots \\
z_{r}(k-h) \\
z
\end{array}\right](1-p \underbrace{1, \ldots, 1}_{(r)}, 0): \\
& \left.\left(a_{j}^{(1)}, \alpha_{j}^{(1)}\right)_{1, P_{1}} ; \cdots ;\left(a_{j}^{(r)}, \alpha_{j}^{(r)}\right)_{1, P_{r}} ;(1-\sigma, 1),\left(a_{j}, \alpha_{j}\right)_{1, P}\right] \\
& \left.\left(b_{j}^{(1)}, \beta_{j}^{(1)}\right)_{1, Q_{1}} ; \cdots ;\left(b_{j}^{(r)}, \beta_{j}^{(r)}\right)_{1, Q_{r}} ;\left(b_{j}, \beta_{j}\right)_{1, Q}\right):
\end{aligned}
$$

and the following conditions are satisfied:

(i) $p+\sum_{i=1}^{r} \min _{1 \leq j \leq M_{i}}\left(b_{j}^{(i)} / \beta_{j}^{(i)}\right)>0$

(ii) $\sigma+\min _{1 \leq j \leq M}\left(b_{j} / \beta_{j}\right)>0$

(iii) The parameters involved in (3.1) are so restricted that $f(x)$ remains positive for $h \leq x \leq k$.

It is not out of place to mention here that the statistical distributions involving Fox's H-function in the p.d.f were studied earlier by Mathai and Saxena [4,5], Srivastava and Singhal [19], Saxena and Dash [8] (see also Srivastava and Kashyap [16] and Springer [10]).

Due to the presence of Fox's H-function, the distribution considered in (3.1) is quite general in nature and a number of classical statistical distributions can be obtained from it on suitably specializing the various parameters involved therein. 
Now, we derive some results for this distribution, with the help of the results obtained in previous sectioin.

It can easily be verified with the help of definition (1.1) and Result 2 that (3.1) represents a p.d.f. i.e. $\int_{-\infty}^{\infty} f(x) d x=1$.

\section{Mathematical Expectation of a Function}

For any function $g(x)$, the expectation of $g(x)$ with respect to p.d.f. $f(x)$ is defined by

$$
E\{g(x)\}=\int_{-\infty}^{\infty} f(x) g(x) d x
$$

Let us consider the function $g(x)$ of the form

$$
g(x)=\prod_{\ell=1}^{s}\left\{\begin{array}{l|l}
H_{p_{\ell^{\prime}}, Q_{\ell^{\prime}}}^{M_{\ell^{\prime}}, N_{\ell^{\prime}}}
\end{array}\left[\begin{array}{l|l}
z_{\ell^{\prime}}(x-h) & \begin{array}{l}
\left(e_{j}^{(\ell)}, E_{j}^{(\ell)}\right)_{1, P_{\ell \prime}} \\
\left(f_{j}^{(\ell)}, F_{j}^{(\ell)}\right)_{1, Q_{\ell^{\prime}}}
\end{array}
\end{array}\right\}\right\}
$$

Taking the p.d.f. $f(x)$ as given by (3.1), using (1.1) and Result 2 of previous section, we then arrive at the following after a little simplification.

$$
\begin{aligned}
& E\{g(x)\}=\frac{u^{\sigma+p-1}}{\triangle} H_{1,1: P_{1} Q_{1} ; \ldots ; P_{r \prime}, Q_{r} ; P_{1} \prime, Q_{1} ; \ldots ; P_{s} \prime, Q_{s} \prime ; P+1,0}^{0,1: M_{1} N_{1} ; \ldots ; M_{r l}, N_{r} ; M_{1} l, N_{1} / \ldots ; M_{s} l, N_{s} l ; M, N+1}
\end{aligned}
$$

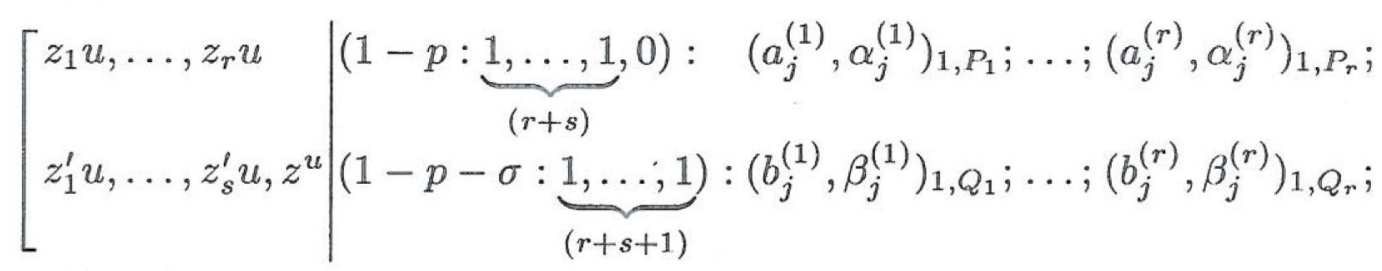

$$
\begin{aligned}
& \left.\left(e_{j}^{(1)}, E_{j}^{(1)}\right)_{1, P_{1}} ; \ldots ;\left(e_{j}^{(s)}, E_{j}^{(s)}\right)_{1, P_{s}^{\prime}} ;(1-\sigma, \mathbb{1}),\left(a_{j}, \alpha_{j}\right)_{1, P}\right] \\
& \left(f_{j}^{(1)}, F_{j}^{(1)}\right)_{1, Q_{1}^{\prime}} ; \ldots ;\left(f_{j}^{(s)}, F_{j}^{(s)}\right)_{1, Q_{s}^{\prime}} ;\left(b_{j}, \beta_{j}\right)_{1, Q}
\end{aligned}
$$

provided that, $\sigma>0, h \neq k$

$$
p+\sum_{i=1}^{r} \min _{1 \leq j \leq M_{i}}\left(b_{j}^{(i)} / \beta_{j}^{(i)}\right)+\sum_{\ell=1}^{s} \min _{1 \leq j \leq M_{i}^{\prime}}\left(f_{j}^{(\ell)} / F_{j}^{(\ell)}\right)>0
$$

and the conditions (ii) and (iii) of (3.3) are satisfied.

\section{Characteristic Function}

The characteristic function $\phi(t)$ of a random variable $x$ with respect to p.d.f. $f(x)$ is defined by

$$
\phi(t)=E\left\{e^{\omega t x}\right\}=\int_{-\infty}^{\infty} e^{\omega t x} f(x) d x, \omega=\sqrt{-1}
$$


Taking the p.d.f. $f(x)$ as given by (3.1), substituting $x$ for $(x-h)$, interpreting the result thus obtained in the operator form with the help of (1.1) and using a paritcular case of Result 3 , we easily arrive at the following

$$
\begin{aligned}
& \phi(t)=\frac{e^{\omega t h} u^{\sigma+p-1}}{\triangle} H_{1,1: P_{1} Q_{1} ; \ldots ; P_{r}, Q_{r} ; 0,1 ; P+1, Q}^{0,1: M_{1} N_{1} ; \ldots ; M_{r l}, N_{r} ; 1,0 ; M, N+1}\left[\begin{array}{l|l}
z_{1} u \\
\vdots \\
z_{r} u \\
-\omega t u \\
z
\end{array} \mid(1-p-\sigma \underbrace{1, \ldots, 1}_{(r+1)}, 0):\right. \\
& \left(a_{j}^{(1)}, \alpha_{j}^{(1)}\right)_{1, P_{1}} ; \ldots ;\left(a_{j}^{(r)}, \alpha_{j}^{(r)}\right)_{1, P_{r}} ; \ldots ; \quad(1-\sigma, 1),\left(a_{j}, \alpha_{j}\right)_{1, P} \\
& \left(b_{j}^{(1)}, \beta_{j}^{(1)}\right)_{1, Q_{1}} ; \ldots ;\left(b_{j}^{(r)}, \beta_{j}^{(r)}\right)_{1, Q_{r}} ;(0,1) ;\left(b_{j}, \beta_{j}\right)_{1, Q}
\end{aligned}
$$

where $u=k-h, \triangle$ is defined by (3.2) and the conditions (3.3) are satisfied.

\section{Distribution Function}

The distribution function (or the commulatice probability function) $F(t)$ for the p.d.f. $f(x)$ is given by

$$
F(t)=\int_{-\infty}^{t} f(x) d x=\int_{h}^{t} f(x) d x
$$

Substituting the value of $f(x)$ from (3.1), expressing the Fox's H-functions in series form $[14$, p.12, eq.(2.2.4)], interchanging the order of integration and summatioins, substituting $(x-h)=(k-h) z$ in the resulting integral and evaluating the integral with the help of $[1, \mathrm{p} .87$, eq.(2.5.3)], we arrive at the following result expressed in terms of $\mathrm{H}$-function of $(r+2)$ variables, after a little simplification

$$
\begin{aligned}
& F(t)=\frac{1}{\triangle} \frac{(t-h)^{p}(k-t)^{\sigma}}{(k-h)} H_{2,2: P_{1} Q_{1} ; \ldots ; P_{r^{\prime}}, Q_{r} ; P, Q ; 1,1}^{0,2: M_{1} N_{1} ; \ldots ; M_{r^{\prime}}, N_{r} ; M, N ; 1,1}\left[z_{1}(t-h), \ldots\right. \\
& z_{r}(t-h), z\left(\frac{k-t}{k-h}\right) \frac{-(t-h)}{k-h} \mid \begin{array}{l}
(1-p: \underbrace{1, \ldots, 1}_{(r)}, 0,0),(1-p-\sigma: \underbrace{1, \ldots, 1}_{(r+2)}): \\
(-p: \underbrace{1, \ldots, 1}_{(r)}, 0,1),(1-p-\sigma: \underbrace{1, \ldots, 1}_{(r+1)}, 0):
\end{array} \\
& \left.\left(a_{j}^{(1)}, \alpha_{j}^{(1)}\right)_{1, P_{1}} ; \ldots ;\left(a_{j}^{(r)}, \alpha_{j}^{(r)}\right)_{1, P_{r}} ;\left(a_{j}, \alpha_{j}\right)_{1, P} ;(0,1)\right] \\
& \left.\left(b_{j}^{(1)}, \beta_{j}^{(1)}\right)_{1, Q_{1}} ; \ldots ;\left(b_{j}^{(r)}, \beta_{j}^{(r)}\right)_{1, Q_{r}} ;\left(b_{j}, \beta_{j}\right)_{1, Q} ;(0,1)\right]
\end{aligned}
$$


where $\triangle$ is defined by (3.2) and the conditions (i) and (iii) of (3.3) are satisfied.

It may be mentioned here that the results obtained in sections (2) to (6) above are quite general in nature and are thus capable of yielding several new or known result. For example, the results obtained earlier by Saigo and Raina [6] and the results discussed in Exton [2, pp.219-240] can be obtained on suitably specializing the results presented in this paper. We, however, omit the details for lack of space.

\section{Acknowledgements}

The authors are thankful to Professor H. M. Srivastava for his encourgement and useful suggestions which led to the present form of the paper.

\section{References}

[1] A. Erdělyi, W. Magnus, F. Oberhettinger and F. G. Tricomi, Higher Transcendental Functions, Vol.I, McGrae-Hill, New York, Toronto and London, 1953.

[2] H. Exton, Multiple Hypergeometric Functions and Applications, Ellis Horwood Ltd., Chichester; Halsted press (John Wiley and Sons), New York, London, Sydney and Toronto, 1976.

[3] C. Fox, "The $\mathrm{G}$ and $\mathrm{H}$ functions as symmetrical Fourier kernels," Trans. Amer. Math. Soc. 98 (1961), 395-429.

[4] A. M. Mathai and R. K. Saxena, "Applications of special functions in the characterization of probability distributions," S. Afr. Statist. J. 3 (1969), 27-34.

[5] A. M. Mathai and R. K. Saxena, "On linear combinations of stochastic variables, Metrika 20 (1973), 160-169.

[6] M. Saigo and R. K. Raina, "On the fractional calculus operator involving Gauss's series and its applications to certain statistical distributions," Rev. Técn. Fac. Ingr. Univ. Zulia, 41 (1991), 53-62.

[7] Sumil Saxena, Investigations in Multivariable Generalized Hypergeometric Functions, Integral Transforms, Fractional Integral Operators and General Class of Polynomials with Applications, Ph. D. Thesis, Univ. of Rajasthan, Jaipur, 1995.

[8] R. I. Saxena and S. P. Dash, "The distribution of the linear combination and the ratio of products of independent random variables associated with an H-function," Vijnana Parishad Anusandhan Patrika, 22 (1979), 57-65.

[9] R. K. Saxena and R. K. Kumbhat, "Integral operators involving H-function," Indian J. Pure Appl. Math. 5 (1974), 1-6.

[10] M. D. Springer, The Algebra of Random Variables, Wiley, New York, 1979.

[11] H. M. Srivastava, "A contour integral involving Fox's H-function," Indian J. Math. 14 $(1974), 1-6$.

[12] H. M. Srivastava, R. C. S. Chandel and P. K. Vishwakarma, "Fractional derivatives of certain generalized hypergeometric functions of several variables," J. Math. Anal. Appl. (3) 184 (1994), 560-572.

[13] H. M. Srivastava and S. P. Goyal, "Fractional derivatives of the H-function of several variables," J. Mat. Anal. Appl. 112 (1985), 641-651.

[14] H. M. Srivastava, K. C. Gupta and S. P. Goyal, The H-Functions of One and Two Variables with Applications, South Asian Publishers, New Delhi and Madras, 1982. 
[15] H. M. Srivastava and M. A. Hussain, "Fractional integration of the H-function of several variables," Computer Math. Applic. (9) 30 (1995), 73-85.

[16] H. M. Srivastava and B. R. K. Kashyap. Special Functions in Queuing Theory and Related Stochastic Processes, Academic Press, New York and London, 1982.

[17] H. M. Srivastava and R. Panda, "Some bilateral generating functions for a class of generalized hypergeometric polynomials," J. Reine Angew. Math. 283/284 (1976), 265-274.

[18] H. M. Srivastava and N. P. Singh, "The integration of certain products of the multivariable H-function with a genral calss of polynomials," Rend. Circ. Mat. Palermo (2) 32 (1983), 157-187.

[19] H. M. Srivastava and J. P. Singhal, "On a class of generalized hypergeometric distributions," Jñánábha Sect. A2 (1972), 1-9.

Department of Mathematics, University of Rajasthan, Jaipur 302004, Rajasthan, India. 